ISSN 1112-9867

http://www.jfas.info

\title{
KSOR ITERATIVE METHOD WITH QUADRATURE SCHEME FOR SOLVING SYSTEM OF FREDHOLM INTEGRAL EQUATIONS OF SECOND KIND
}

\author{
N. Z. F. M. Radzuan, M. N. Suardi and J. Sulaiman* \\ Mathematics with Economics Programme, Universiti Malaysia Sabah, 88400 Kota Kinabalu, \\ Sabah, Malaysia
}

Published online: 17 October 2017

\begin{abstract}
In this study, the system of second kind Fredholm integral equations has been discretized by using the first order quadrature scheme namely trapezoidal rule in order to construct the first order quadrature approximation equation. Next, the quadrature approximation equation obtained has been used to construct a system of linear equations. Three types of iterative methods were used to solve the system of linear equations such as Gauss-Seidel (GS), Successive Over Relaxation (SOR) and Kaudd Successive Over Relaxation (KSOR). For comparison purpose, two problems have been considered in this study in order to analyze the efficiency of these three proposed iterative methods for solving the problems. Based on the numerical results, it can be pointed out that KSOR is similar as SOR but both of these iterative methods are more efficient than GS method.
\end{abstract}

Keywords: quadrature scheme; system of Fredholm integral equations; KSOR iterative method.

Author Correspondence, e-mail: jumat@ums.edu.my

doi: http://dx.doi.org/10.4314/jfas.v9i5s.43 


\section{INTRODUCTION}

System of Fredholm integral equations is one of a family of integral equations (IEs). This system has a fixed integral area since the limit of integral is constant. This system has two or more equations in the set. System of second kind Fredholm integral equations generally can be defined as

$$
y_{r}(x)=F_{r}(x)+\sum_{s=1}^{m} \int_{a}^{b} k_{r s}(x, t) y_{s}(t) d t
$$

where $y_{s}(t)$ is an unknown function, $K_{r s}(x, t)$ is a Kernel function, $y_{r}(x)$ is a known function, $F_{r}$ and $K_{r s}$ are continuous functions [1].

System of Fredholm integral equations is often associated with the boundary value problem [2]. This system also represent many physical problems related to science and engineering problem. Therefore, many numerical methods being used to solve the problems of the system of integral equations. For example, the methods have been used such as the domain decomposition method [3]; the Open Newton-Cotes Formula [1] and the method of collocation with Legendre polynomial [4]. Furthermore, these problems have also been solved using Bernstein polynomial [5] and new algorithm developed by [6]. However, the main objective of this paper deals with the application of the KSOR iteration with the first order quadrature discretization scheme for solving the system of second kind Fredholm integral equations.

\section{QUADRATURE APPROXIMATION EQUATION}

In this section, the discretization process is necessary to produce the quadrature approximation equation to construct system of linear equations. However, this paper proposes discretization of problem (1) by using the first order of quadrature scheme, trapezoidal rule. Prior to that, consider the generally quadrature scheme as follows

$$
\int_{a}^{b} y(t) d t=\sum_{j=0}^{n} A_{j} y\left(t_{j}\right)+\varepsilon_{n}(y)
$$

where $t_{j}, A_{j}$ and $\varepsilon_{n}(y)$ are the quadrature point in the interval $[a, b]$, weights quadrature and error respectively [7]. By considering the trapezoidal rule which is known as the first order quadrature scheme, let the interval $[a, b]$ be divided into several sets $\left\{x_{0}, x_{1}, x_{2}, \ldots, x_{n}\right\}$ with the number of $(n)$ subintervals of equal width, $h_{\tau}$ in which $h_{\tau}$ is defined as 


$$
h_{\tau}=\frac{b-a}{(n)}
$$

Fig. 1 shows the finite grid network that is used to construct the quadrature approximation equations where the GS, SOR and KSOR iterative methods are applied onto each interior node points until the iteration is converged.

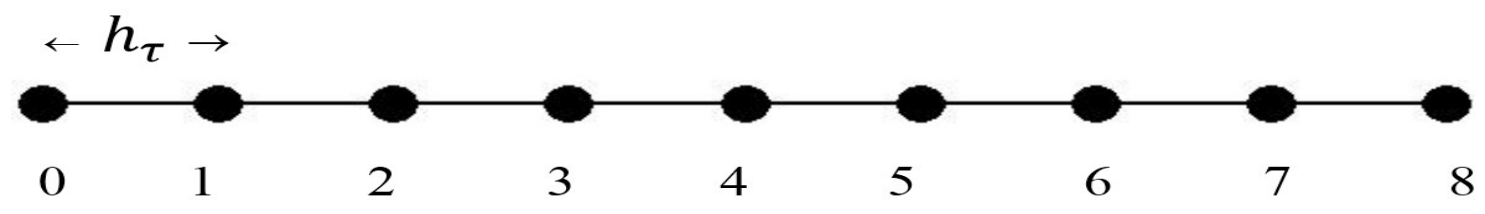

Fig.1. The uniformly distribution of interior node quadrature points for the solution domain

$$
[a, b] \text { at } n=8
$$

Based on Fig. 1 and consider the set $\left\{x_{0}, x_{1}, x_{2}, \ldots, x_{n}\right\}$, then the integral function $F(x)$ on the interval $[a, b]$ can be expressed as follows

$$
\int_{a}^{b} F(x) d x=\int_{x_{i}}^{x_{n}} F(x) d x
$$

For the purpose of illustration, let $a=0, b=8$ and $i=0,1,2, \ldots, n$ then Equation (3) can be rewritten as

$$
\begin{gathered}
\int_{0}^{8} F(x) d x=\int_{x_{0}}^{x_{1}} F(x) d x+\int_{x_{1}}^{x_{2}} F(x) d x+\int_{x_{2}}^{x_{3}} F(x) d x+\int_{x_{3}}^{x_{4}} F(x) d x+ \\
\int_{x_{4}}^{x_{5}} F(x) d x+\int_{x_{5}}^{x_{6}} F(x) d x+\int_{x_{6}}^{x_{7}} F(x) d x+\int_{x_{7}}^{x_{8}} F(x) d x
\end{gathered}
$$

$F(x)$

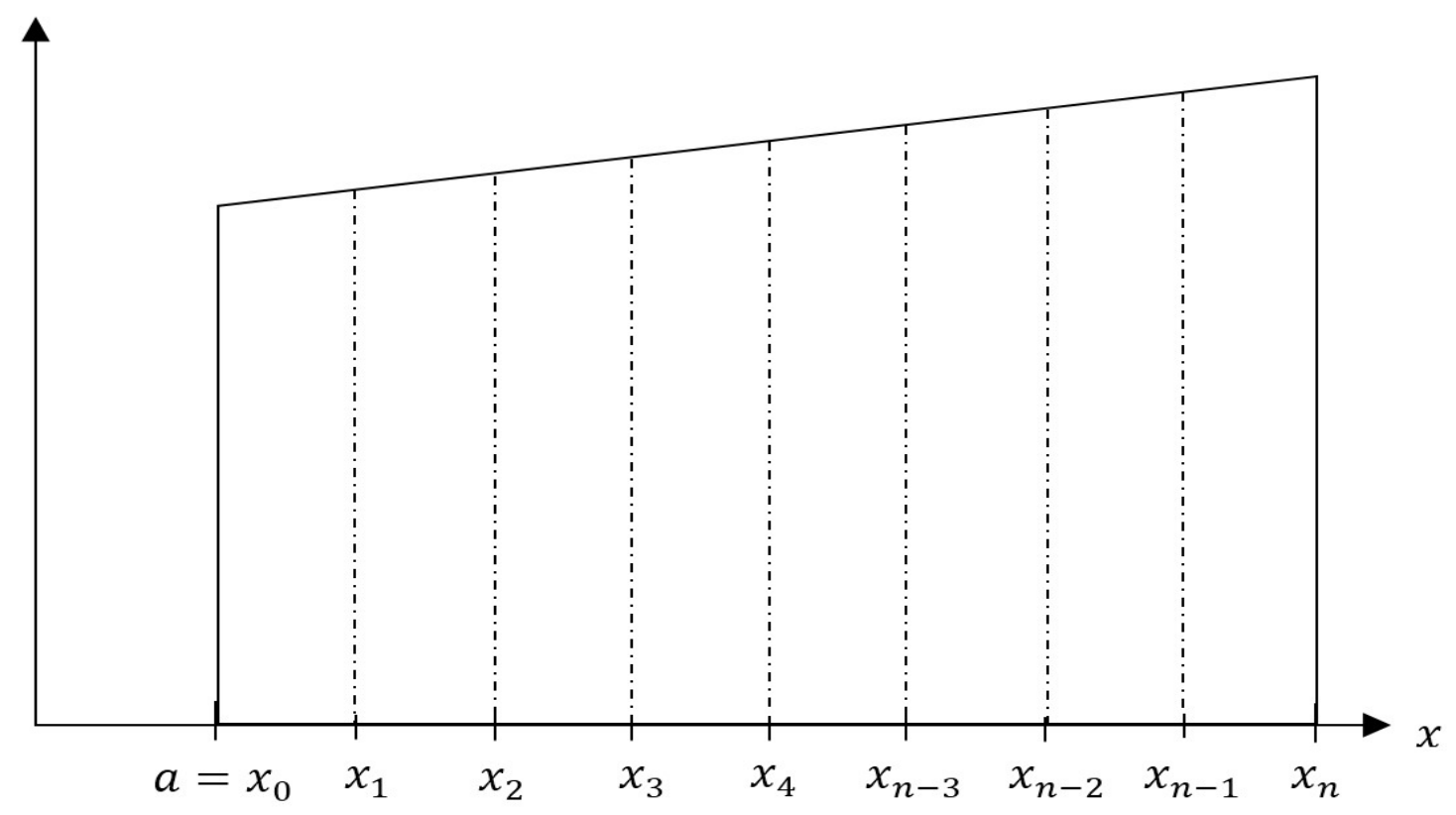

Fig.2.The definite integral of function, $F(x)$ over the interval $[a, b]$ at $n=8$ 
Based on Fig. 2 and by applying the trapezium scheme over Equation (4), it can be obtained as

$$
\int_{0}^{8} F(x) d x=\frac{h_{\tau}}{2} F_{0}+h_{\tau} F_{1}+h_{\tau} F_{2}+h_{\tau} F_{3}+h_{\tau} F_{4}+h_{\tau} F_{5}+h_{\tau} F_{6}+h_{\tau} F_{7}+\frac{h_{\tau}}{2} F_{8}
$$

From Equation (5), the weights quadrature coefficient, $A_{j}$ in Equation (2) can be defined as

$$
A_{j}= \begin{cases}\frac{1}{2} h_{\tau}, & j=0, n \\ h_{\tau}, & j=1,2, \ldots, n-1\end{cases}
$$

Let the error, $\varepsilon_{n}(y)$ in Equation (2) be ignored and then consider Equation (1) with $r=$ 1,2and $m=2$, two equations can be formed to construct a system of second kind Fredholm integral equations which can be defined as

$$
\begin{aligned}
& y_{1}(x)=F_{1}(x)+\int_{a}^{b} K_{11}(x, t) y_{1}(t) d t+\int_{a}^{b} K_{12}(x, t) y_{2}(t) d t \\
& y_{2}(x)=F_{2}(x)+\int_{a}^{b} K_{21}(x, t) y_{1}(t) d t+\int_{a}^{b} K_{22}(x, t) y_{2}(t) d t
\end{aligned}
$$

By imposing Equation (5) into Equations (7a) and (7b), the trapezium approximation equations for a system of integral equations can be used to construct in the following approximation equations

$$
\begin{aligned}
& y_{1}(x)-\left(\frac{h_{\tau}}{2} K_{11}\left(x, t_{0}\right) y_{1,0}+h_{\tau} K_{11}\left(x, t_{1}\right) y_{1,1}+h_{\tau} K_{11}\left(x, t_{2}\right) y_{1,2}+h_{\tau} K_{11}\left(x, t_{3}\right) y_{1,3}\right. \\
& +h_{\tau} K_{11}\left(x, t_{4}\right) y_{1,4}+h_{\tau} K_{11}\left(x, t_{5}\right) y_{1,5}+h_{\tau} K_{11}\left(x, t_{6}\right) y_{1,6} \\
& +h_{\tau} K_{11}\left(x, t_{7}\right) y_{1,7}+ \\
& \frac{h_{\tau}}{2} K_{11}\left(x, t_{8}\right) y_{1,8}+\frac{h_{\tau}}{2} K_{12}\left(x, t_{0}\right) y_{2,0}+h_{\tau} K_{12}\left(x, t_{1}\right) y_{2,1}+h_{\tau} K_{12}\left(x, t_{2}\right) y_{2,2}+ \\
& h_{\tau} K_{12}\left(x, t_{3}\right) y_{2,3}+h_{\tau} K_{12}\left(x, t_{4}\right) y_{2,4}+h_{\tau} K_{12}\left(x, t_{5}\right) y_{2,5}+h_{\tau} K_{12}\left(x, t_{6}\right) y_{2,6}+ \\
& \left.h_{\tau} K_{12}\left(x, t_{7}\right) y_{1,7}+\frac{h_{\tau}}{2} K_{12}\left(x, t_{8}\right) y_{2,8}\right)=F_{1}(x) \\
& y_{2}(x)-\left(\frac{h_{\tau}}{2} K_{21}\left(x, t_{0}\right) y_{1,0}+h_{\tau} K_{21}\left(x, t_{1}\right) y_{1,1}+h_{\tau} K_{21}\left(x, t_{2}\right) y_{1,2}+h_{\tau} K_{21}\left(x, t_{3}\right) y_{1,3}\right. \\
& +h_{\tau} K_{21}\left(x, t_{4}\right) y_{1,4}+h_{\tau} K_{21}\left(x, t_{5}\right) y_{1,5}+h_{\tau} K_{21}\left(x, t_{6}\right) y_{1,6} \\
& +h_{\tau} K_{21}\left(x, t_{7}\right) y_{1,7}+
\end{aligned}
$$




$$
\begin{aligned}
\frac{h_{\tau}}{2} K_{21}\left(x, t_{8}\right) y_{1,8} & +\frac{h_{\tau}}{2} K_{22}\left(x, t_{0}\right) y_{2,0}+h_{\tau} K_{22}\left(x, t_{1}\right) y_{2,1}+h_{\tau} K_{22}\left(x, t_{2}\right) y_{2,2} \\
+ & h_{\tau} K_{22}\left(x, t_{3}\right) y_{2,3}+h_{\tau} K_{22}\left(x, t_{4}\right) y_{2,4}+h_{\tau} K_{22}\left(x, t_{5}\right) y_{2,5} \\
+ & h_{\tau} K_{22}\left(x, t_{6}\right) y_{2,6}+ \\
h_{\tau} K_{22}\left(x, t_{7}\right) y_{1,7}+\frac{h_{\tau}}{2} & \left.K_{22}\left(x, t_{8}\right) y_{2,8}\right)=F_{2}(x)
\end{aligned}
$$

Furthermore, the approximate Equations (8a) and (8b) can be manipulated to form a linear system in matrix form generally as

$$
K \underline{y}=\underline{F}
$$

where

$$
\begin{aligned}
& K=\left[\begin{array}{ll}
K_{A} & K_{B} \\
K_{C} & K_{D}
\end{array}\right] \\
& y=\left[\begin{array}{lllllll}
y_{1,0} y_{1,1} & y_{1,2} & \ldots & y_{1, n-1} & y_{1, n} y_{2,0} y_{2,1} y_{2,2} & \ldots & y_{2, n-1} y_{2, n}
\end{array}\right]^{T}, \\
& \underline{F}=\left[\begin{array}{lllllllll}
f_{1,0} f_{1,1} & f_{1,2} & \ldots & f_{1, n-1} & f_{1, n} & f_{2,0} & f_{2,1} f_{2,2} & \ldots & f_{2, n-1} f_{2, n}
\end{array}\right]^{T} .
\end{aligned}
$$

Meanwhile, $K_{A}, K_{B}, K_{C}$ and $K_{D}$ are submatrices which are defined as follows $K_{A}=$

$$
\left[\begin{array}{ccccc}
1-\frac{h_{\tau}}{2} K_{11}(0,0) & -h_{\tau} K_{11}(0,1) & -h_{\tau} K_{11}(0,2) & \cdots & -\frac{h_{\tau}}{2} K_{11}(0, n) \\
-\frac{h_{\tau}}{2} K_{11}(1,0) & 1-h_{\tau} K_{11}(1,1) & -h_{\tau} K_{11}(1,2) & \cdots & -\frac{h_{\tau}}{2} K_{11}(1, n) \\
-\frac{h_{\tau}}{2} K_{11}(2,0) & -h_{\tau} K_{11}(2,1) & 1-h_{\tau} K_{11}(2,2) & \cdots & -\frac{h_{\tau}}{2} K_{11}(2, n) \\
\vdots & \vdots & \vdots & & \vdots \\
-\frac{h_{\tau}}{2} K_{11}(n, 0) & -h_{\tau} K_{11}(n, 1) & -h_{\tau} K_{11}(n, 2) & \cdots & 1-\frac{h_{\tau}}{2} K_{11}(n, n)
\end{array}\right]
$$

$K_{B}=$

$$
\left[\begin{array}{ccccc}
-\frac{h_{\tau}}{2} K_{12}(0,0) & -h_{\tau} K_{12}(0,1) & -h_{\tau} K_{12}(0,2) & \cdots & -\frac{h_{\tau}}{2} K_{12}(0, n) \\
-\frac{h_{\tau}}{2} K_{12}(1,0) & -h_{\tau} K_{12}(1,1) & -h_{\tau} K_{12}(1,2) & \cdots & -\frac{h_{\tau}}{2} K_{12}(1, n) \\
-\frac{h_{\tau}}{2} K_{12}(2,0) & -h_{\tau} K_{12}(2,1) & -h_{\tau} K_{12}(2,2) & \cdots & -\frac{h_{\tau}}{2} K_{12}(2, n) \\
\vdots & \vdots & \vdots & & \vdots \\
-\frac{h_{\tau}}{2} K_{12}(n, 0) & -h_{\tau} K_{12}(n, 1) & -h_{\tau} K_{12}(n, 2) & \cdots & -\frac{h_{\tau}}{2} K_{12}(n, n)
\end{array}\right]
$$




$$
\begin{aligned}
& {\left[\begin{array}{ccccc}
-\frac{h_{\tau}}{2} K_{21}(0,0) & -h_{\tau} K_{21}(0,1) & -h_{\tau} K_{21}(0,2) & \cdots & -\frac{h_{\tau}}{2} K_{21}(0, n) \\
-\frac{h_{\tau}}{2} K_{21}(1,0) & -h_{\tau} K_{21}(1,1) & -h_{\tau} K_{21}(1,2) & \cdots & -\frac{h_{\tau}}{2} K_{21}(1, n) \\
-\frac{h_{\tau}}{2} K_{21}(2,0) & -h_{\tau} K_{21}(2,1) & -h_{\tau} K_{21}(2,2) & \cdots & -\frac{h_{\tau}}{2} K_{21}(2, n) \\
\vdots & \vdots & \vdots & & \vdots \\
-\frac{h_{\tau}}{2} K_{21}(n, 0) & -h_{\tau} K_{21}(n, 1) & -h_{\tau} K_{21}(n, 2) & \cdots & -\frac{h_{\tau}}{2} K_{21}(n, n)
\end{array}\right]} \\
& K_{D}= \\
& {\left[\begin{array}{ccccc}
1-\frac{h_{\tau}}{2} K_{22}(0,0) & -h_{\tau} K_{22}(0,1) & -h_{\tau} K_{22}(0,2) & \cdots & -\frac{h_{\tau}}{2} K_{22}(0, n) \\
-\frac{h_{\tau}}{2} K_{22}(1,0) & 1-h_{\tau} K_{22}(1,1) & -h_{\tau} K_{22}(1,2) & \cdots & -\frac{h_{\tau}}{2} K_{22}(1, n) \\
-\frac{h_{\tau}}{2} K_{22}(2,0) & -h_{\tau} K_{22}(2,1) & 1-h_{\tau} K_{22}(2,2) & \cdots & -\frac{h_{\tau}}{2} K_{22}(2, n) \\
\vdots & \vdots & \vdots & & \vdots \\
-\frac{h_{\tau}}{2} K_{22}(n, 0) & -h_{\tau} K_{22}(n, 1) & -h_{\tau} K_{22}(n, 2) & \cdots & 1-\frac{h_{\tau}}{2} K_{22}(n, n)
\end{array}\right] .}
\end{aligned}
$$

Clearly, $K, y$ and $f$ are known as the coefficient matrix, unknown vector and known vector respectively.

\section{DERIVATION OF PROPOSED ITERATION SCHEMES}

By referring to the system of linear equations in Equation (9), this linear system will be solved iteratively by using GS, SOR and KSOR iterative methods. These three iterative methods can be classified as a family of point iteration methods.

\subsection{GS Iteration Scheme}

Since implementations of these three iterative methods based on the point iteration approach, we need to consider again the coefficient matrix, $K$ in Equation (9). As we know, the coefficients matrix, $K$ of the linear system (9) can be manipulated to derive for the formulation of different iterative methods. To do this, let the coefficient matrix, $K$ of the system of linear equations (9) be decomposed as

$$
K=L+D+U
$$

where $U, L$ and $D$ is anupper triangular matrix, lower triangular matrix and a diagonal matrix. By using the definition of Equation (10), the linear systems (9) can be rewritten as 


$$
(L+D+U) \underline{y}=\underline{F}
$$

By referring to the linear system (11), the general scheme of the GS iterative method can be stated in matrix form as

$$
\underline{y}^{(k+1)}=(D-L)^{-1} U \underline{y}^{(k)}+(L-D)^{-1} \underline{F}
$$

Or, it can be shown that the formulation of this iteration scheme can be identified in point iteratively as

$$
y_{i}^{(k+1)}=\frac{1}{K_{i i}}\left(F_{i}-\sum_{j=1}^{i-1} K_{i j} y_{j}^{(k+1)}-\sum_{j=i+1}^{n} K_{i j} y_{j}^{(k)}\right)
$$

By referring to Fig. 1 and Equation (13), algorithm 1 shows the implementation of GS iterative method.

Algorithm 1: GS scheme

i. Set initial value $y^{(0)}=0$.

ii. Calculate the coefficient matrix, $K$.

iii. Calculate the vector, $\underline{F}$.

iv. For $i=0,1,2, \ldots, n$, calculate

$$
y_{i}^{(k+1)}=\frac{1}{K_{i i}}\left(F_{i}-\sum_{j=1}^{i-1} K_{i j} y_{j}^{(k+1)}-\sum_{j=i+1}^{n} K_{i j} y_{j}^{(k)}\right)
$$

v. Check the convergence test, $\left|y_{i}^{(k+1)}-y_{i}^{(k)}\right|<\varepsilon=10^{-10}$. If yes, go to step (vi).

Otherwise, go back to step (iv).

vi. Display numerical solution.

\subsection{SOR Iteration Scheme}

To improve the convergence of the GS iterative method, SOR iterative method introduced by Young in [8-11] is the same as GS iterative method but the use of the relaxation parameter, $\omega$ is able to accelerate convergence and reduce error rates approximate solution. The most suitable range for the value of $\omega$ is 0 to 2 [12]. The ability of the SOR iterative method is shown to have a rapid rate of convergence solutions such as in [8-11, 13-14]. Generally, the SOR iteration scheme has the form as [15]

$$
y_{i}^{(k+1)}=(1-\omega) y_{i}^{(k)}+\frac{\omega}{K_{i i}}\left(F_{i}-\sum_{j=1}^{i-1} K_{i j} y_{j}^{(k+1)}-\sum_{j=i+1}^{n} K_{i j} y_{j}^{(k)}\right)
$$


where $i=0,1,2, \ldots, n$.

Based on Equation (14), algorithm 2 describes the implementation of SOR iterative method. As taking $\omega=1$, this iteration scheme can be recognized as the standard GS iterative method.

Algorithm 2: SOR scheme

i. Set initial value $y^{(0)}=0$.

ii. Calculate the coefficient matrix, $K$.

iii. Calculate the vector, $\underline{F}$.

iv. For $i=0,1,2, \ldots, n$, calculate

$$
y_{i}^{(k+1)}=(1-\omega) y_{i}^{(k)}+\frac{\omega}{K_{i i}}\left(F_{i}-\sum_{j=1}^{i-1} K_{i j} y_{j}^{(k+1)}-\sum_{j=i+1}^{n} K_{i j} y_{j}^{(k)}\right)
$$

v. Check the convergence test, $\left|y_{i}^{(k+1)}-y_{i}^{(k)}\right|<\varepsilon=10^{-1}$. If yes, go to step (vi). Otherwise, go back to step (iv).

vi. Display numerical solution.

\subsection{KSOR Iteration Scheme}

The KSOR iterative method is a new variant of SOR method that was introduced by [16]. The advantage of this is able to update the first component in the first equation of the first step that reflects the rapid convergence at the beginning [16]. Relaxation parameter, $\omega$ for KSOR method has a sensitivity that is less than the SOR [17]. The general formula of the KSOR iteration scheme can be stated as

$$
y_{i}^{(k+1)}=y_{i}^{(k)}+\frac{\omega}{K_{i i}}\left(\begin{array}{c}
F_{i}-\sum_{j=1}^{i-1} K_{i j} y_{j}^{(k+1)}-\sum_{j=i+1}^{n} K_{i j} y_{j}^{(k)} \\
-K_{i j} y_{j}^{(k+1)}
\end{array}\right)
$$

where $i=0,1,2, \ldots, n$. According to Equation (15), the implementation of KSOR iteration scheme may be elaborated in algorithm 3 .

Algorithm 3: KSOR scheme

i. Set initial value $y^{(0)}=0$.

ii. Calculate the coefficient matrix, $K$.

iii. Calculate the vector, $\underline{F}$.

iv. For $i=0,1,2, \ldots, n$, calculate 


$$
y_{i}^{(k+1)}=y_{i}^{(k)}+\frac{\omega}{K_{i i}}\left(\begin{array}{c}
F_{i}-\sum_{j=1}^{i-1} K_{i j} y_{j}^{(k+1)}-\sum_{j=i+1}^{n} K_{i j} y_{j}^{(k)} \\
-K_{i j} y_{j}^{(k+1)}
\end{array}\right)
$$

v. Check the convergence test, $\left|y_{i}^{(k+1)}-y_{i}^{(k)}\right|<\varepsilon=10^{-10}$. If yes, go to step (vi). Otherwise, go back to step (iv).

vi. Display numerical solution.

\section{NUMERICAL EXPERIMENTS}

To evaluate the performance of these three iterative methods such as GS, SOR and KSOR, there are two examples of system of second kind Fredholm integral equations were considered to be test problems. For the purpose of comparison between these three iterative methods, there are three parameters such as number of iterations (Iter), computational time in seconds (Time) and maximum error (Error) will be recorded in the table form. In addition to that, the tolerance error, $\varepsilon$ considered in the implementation of point iteration based on these three proposed iterative methods is $\varepsilon=10^{-10}$.

\subsection{Problem 1 [4]}

$$
\begin{aligned}
& y_{1}(x)=\frac{x}{18}+\frac{17}{36}+\int_{0}^{1} \frac{(x+t)}{3}\left(y_{1}(t)+y_{2}(t)\right) d t \\
& y_{2}(x)=x^{2}-\frac{19}{12} x+1+\int_{0}^{1} x t\left(y_{1}(t)+y_{2}(t)\right) d t
\end{aligned}
$$

The exact solutions for the system of Fredholm integral Equation (16) can be given as

$$
\begin{gathered}
y_{1}(x)=x+1 \\
y_{2}(x)=x^{2}+1
\end{gathered}
$$

\subsection{Problem 2 [3]}

$$
\begin{aligned}
& y_{1}(x)=\frac{5}{6} x^{2}-\frac{25}{12} x+1+\int_{0}^{1} x(1+t) y_{1}(t) d t+\int_{0}^{1} x^{2} t y_{2}(t) d t \\
& y_{2}(x)=x^{4}-\frac{1}{5} x^{2}-\frac{7}{12} x+\int_{0}^{1} x t y_{1}(t) d t+\int_{0}^{1}\left(x^{2}-x t\right) y_{2}(t) d t
\end{aligned}
$$

The exact solutions for the system of Fredholm integral Equation (17) can be shown as

$$
\begin{gathered}
y_{1}(x)=x^{2}+1 \\
y_{2}(x)=x^{4}
\end{gathered}
$$

According to two systems of second kind Fredholm integral equations in Equations (16) and 
(17), all the results of numerical experiments were recorded in Tables 1 and 2. The numerical results in Table 3 showed that the SOR iterative method has reduced number of iterations approximately $48.00 \%-77.39 \%$ and computational time approximately $0 \%-81.82 \%$ for solving the systems of second kind Fredholm integral equations when compared to the GS iterative method, whereas the KSOR iterative method has reduced number of iterations approximately $48.00 \%-77.61 \%$ and computational time approximately $0 \%-99.50 \%$. Therefore, it can be pointed out that the SOR and KSOR iterative methods are better than GS iterative method in terms of number of iterations and computational time. 
Table 1. Comparison of number of iterations (Iter), computational time in seconds (Time) and maximum absolute error (Error) on iterative methods for Problem 1

\begin{tabular}{|c|c|c|c|}
\hline \multicolumn{4}{|c|}{ Iter } \\
\hline $\mathbf{M}$ & GS & SOR & KSOR \\
\hline 50 & 196 & $\begin{array}{c}45.0 \\
(w=1.56)\end{array}$ & $\begin{array}{c}45.0 \\
(w=-2.75)\end{array}$ \\
\hline 100 & 199 & $\begin{array}{c}45.0 \\
(w=1.56)\end{array}$ & $\begin{array}{c}45.0 \\
(w=-2.76)\end{array}$ \\
\hline 200 & 201 & $\begin{array}{c}46.0 \\
(w=1.57)\end{array}$ & $\begin{array}{c}45.0 \\
(w=-2.77)\end{array}$ \\
\hline 400 & 202 & $\begin{array}{c}46.0 \\
(w=1.57)\end{array}$ & $\begin{array}{c}46.0 \\
(w=-2.78)\end{array}$ \\
\hline 800 & 202 & $\begin{array}{c}46.0 \\
(w=1.57)\end{array}$ & $\begin{array}{c}46.0 \\
(w=-2.78)\end{array}$ \\
\hline \multicolumn{4}{|c|}{ Time (Second) } \\
\hline $\mathbf{M}$ & GS & SOR & KSOR \\
\hline 50 & 0.11 & 0.02 & 0.02 \\
\hline 100 & 0.11 & 0.03 & 0.03 \\
\hline 200 & 0.33 & 0.08 & 0.08 \\
\hline 400 & 0.81 & 0.20 & 0.20 \\
\hline 800 & 3.03 & 0.69 & 0.68 \\
\hline \multicolumn{4}{|c|}{ Error } \\
\hline $\mathbf{M}$ & GS & SOR & KSOR \\
\hline 50 & $8.2081 \mathrm{e}-01$ & $8.2081 \mathrm{e}-01$ & $8.2081 \mathrm{e}-01$ \\
\hline 100 & $7.8860 \mathrm{e}-01$ & $7.8860 \mathrm{e}-01$ & $7.8860 \mathrm{e}-01$ \\
\hline 200 & $7.7367 e-01$ & $7.7367 \mathrm{e}-01$ & $7.7367 \mathrm{e}-01$ \\
\hline 400 & $7.6651 \mathrm{e}-01$ & $7.6651 \mathrm{e}-01$ & $7.6651 \mathrm{e}-01$ \\
\hline 800 & $7.6299 \mathrm{e}-01$ & $7.6299 \mathrm{e}-01$ & $7.6299 \mathrm{e}-01$ \\
\hline
\end{tabular}


Table 2. Comparison of number of iterations (Iter),computational time in seconds (Time) and maximum absolute error (Error) on iterative methods for Problem 2

\begin{tabular}{cccc}
\hline M & \multicolumn{2}{c}{ Iter } & KSOR \\
\hline 50 & GS & SOR & 17.0 \\
& 33 & $(\mathrm{w}=1.21)$ & $(\mathrm{w}=-5.59)$ \\
100 & 33 & 17.0 & 17.0 \\
200 & 33 & $(\mathrm{w}=1.21)$ & $(\mathrm{w}=-5.68)$ \\
& & 17.0 & 17.0 \\
400 & 33 & 17.0 & $(\mathrm{w}=-5.79)$ \\
& & $(\mathrm{w}=1.21)$ & $(\mathrm{w}=-5.95)$ \\
& 33 & 17.0 & 17.0 \\
& & $(\mathrm{w}=1.21)$ & $(\mathrm{w}=-6.06)$ \\
\hline
\end{tabular}

Time (Second)

\begin{tabular}{cccc} 
M & GS & SOR & KSOR \\
\hline 50 & 0.01 & 0.01 & 0.01 \\
100 & 0.02 & 0.01 & 0.01 \\
200 & 0.06 & 0.03 & 0.03 \\
400 & 0.15 & 0.08 & 0.07 \\
800 & 0.50 & 0.26 & 0.15 \\
\hline
\end{tabular}

\section{Error}

\begin{tabular}{cccc}
$\mathbf{M}$ & GS & SOR & KSOR \\
\hline $\mathbf{5 0}$ & $7.7515 \mathrm{e}-01$ & $7.7515 \mathrm{e}-01$ & $7.7515 \mathrm{e}-01$ \\
$\mathbf{1 0 0}$ & $7.8347 \mathrm{e}-01$ & $7.8347 \mathrm{e}-01$ & $7.8347 \mathrm{e}-01$ \\
$\mathbf{2 0 0}$ & $7.8758 \mathrm{e}-01$ & $7.8758 \mathrm{e}-01$ & $7.8758 \mathrm{e}-01$ \\
$\mathbf{4 0 0}$ & $7.8963 \mathrm{e}-01$ & $7.8963 \mathrm{e}-01$ & $7.8963 \mathrm{e}-01$ \\
$\mathbf{8 0 0}$ & $7.9065 \mathrm{e}-01$ & $7.9065 \mathrm{e}-01$ & $7.9065 \mathrm{e}-01$ \\
\hline
\end{tabular}


Table 3. Depreciation percentage of the number and time of iteration for SOR and KSOR iterative methods compared with GS iterative method

\begin{tabular}{lccc}
\hline & & SOR & KSOR \\
\hline Problem 1 & Iter & $77.04-77.39 \%$ & $77.04-77.61 \%$ \\
& Time & $72.73-81.82 \%$ & $86.36-99.50 \%$ \\
\hline Problem 2 & Iter & $48.00 \%$ & $48.00 \%$ \\
& Time & $0.00-50.00 \%$ & $0.00-70.00 \%$ \\
\hline
\end{tabular}

\section{CONCLUSION}

In this paper, the problems of the system of second kind Fredholm integral equations in Equations (16) and (17) have been successfully discretized by using the first order quadrature scheme to derive the corresponding first order quadrature approximation equation. The linear system generated from this quadrature approximation equation has been solved iteratively via GS, SOR and KSOR iterative methods. From the observation of numerical results in Tables 1, 2 and 3 clearly stated that both SOR and KSOR iterative methods require less iteration number and computational time as compared with GS iterative method. However, the accuracy of these three iterative methods are in a good agreement. Overall, since this paper just considered a family of point iterative methods, future study can be extended to investigate the proposed approximate solutions through block iterative method as discussed in EDG [18], EG [19-20] and AGE [21].

\section{REFERENCES}

[1] Ibraheem G H. Solving system of linear Fredholm integral equations of second kind using Open Newton-Cotes formulas. Ibn Al- Haitham Journal for Pure and Applied Science, 2011, 24(2):1-18

[2] Wazwaz A. M. Linear and nonlinear integral equations: Methods and application. Beijing: Higher Education Press, 2011

[3] Ngarasta N, Rodoumta K, Sosso H. The decomposition method applied to systems of linear Volterra integral equations of the first kind. Kybernetes, 2009, 38(3/4):606-614 
[4] Maleknejad K, Nouri K, Torkzadeh L. Comparison projection method with Adomian'sdecomposition method for solving system of integral equations. Bulletin of the Malaysian Mathematical Sciences Society, 2011, 34(2):379-388

[5] Jafarian A, Nia S A M, Golmankhaneh A K, Baleanu D. Numerical solution of linear integral equations system using the Bernstein collocation method. Advances in Difference Equations, 2013, 2013(123):1-15.

[6] Rasulov A, Kilicman A, Eshkuvatov Z, Raimova G. A new algorithm for system of integral equations. Abstract and Applied Analysis, 2014, 2014:1-12

[7] Muthvalu M S,Sulaiman J. Half-sweep arithmetic mean method to solve linear Fredholmequations. InSimposiumKebangsaanSainsMatematik ke-15, 2007,pp. 211-218

[8] Young DM. Iterative methods for solving partial difference equations of elliptic type.Transaction of the American Mathematical Society, 1954, 76(1):92-111

[9] Young D.M. Iterative solution of large linear systems. London: Academic Press, 1971

[10] Young DM. Second-degree iterative methods for the solution of large linear systems. Journal of Approximation Theory, 1972, 5(2):137-148

[11] Young D. M. Iterative solution of linear systems arising from finite element techniques. In: J. R. Whiteman (Eds.),Themathematics of finite elements and applications II. London: Academic Press, 1976, pp. 439-464

[12] Smith G. D. Numerical solution of partial differential equations: Finite difference methods.Oxford: Clarendon Press, 1985

[13] Hunt D N, Triggs C M. Iterative missing value estimation. Journal of the Royal Statistical Society: Series C (Applied Statistics), 1989, 38(2):293-300

[14] Faires J. D., Burden R. Numerical methods. California: Thomson, 2011

[15] Youssef I K, Meligy S A. Boundary value problem on triangular domains and MKSOR method. Applied and Computation Mathematics, 2014, 3(3):90-99

[16] Youssef I. On the successive overrelaxation method. Journal of Mathematics and Statistics, 2012, 8(2):176-184

[17] Youssef I K, Ibrahim R A. Boundary value problems, Fredholm integral equations, SOR and KSOR methods. Life Science Journal, 2013, 10(2):304-312

[18] Abdullah AR. The four point explicit decoupled group (EDG) method: A fast Poisson 
solver. International Journal Computer Mathematics, 1991, 38(1-2):61-70

[19] Evans D J.Group explicit iterative methods for solving large linear systems. International Journal of Computer Mathematics, 1985, 17(1):81-108

[20] Evans D J, Yousif W S. The explicit block relaxation method as a grid smoother in the multigrid V-cycle scheme. International Journalof Computer Mathematics, 1990, $34(1-2): 71-78$.

[21] Evans D J, SahimiMS. The alternating group explicit iterative method (AGE) to solve parabolic and hyperbolic partial differential equations. Annual Review of Heat Transfer, 1989, 2(2):283-389

\section{How to cite this article:}

Radzuan N Z F M, Suardi M N and Sulaiman J. KSOR Iterative Method with Quadrature Scheme for Solving System of Fredholm Integral Equations of Second Kind. J. Fundam. Appl. Sci., 2017, 9(5S), 609-623. 